\title{
A COMPARISON OF SELECTIVE METHODS AND A TEST OF THE PRE-ADAPTATION HYPOTHESIS
}

\author{
JACK BENNETT * \\ Department of Genetics, $\uparrow$ University of Wisconsin, Madison, Wisconsin
}

Received 23.xii.59

\section{INTRODUCTION}

THE capacity of some species to adapt to poisons introduced into their environment has been known for many years. The work is summarised in the review articles of Crow (1957), Milani (1957), and Brown (1958).

Earlier work with DDT has produced conflicting results on the hereditary basis, and manner of selection, of resistance (Crow, I957; Milani, 1957; Tsukamoto, 1958). Different species of insects seemed to resist the poison by different genetic mechanisms. The same species, viz., Drosophila melanogaster, adapted by quite different hereditary changes in different laboratories under varying conditions of culture and selection. Some resistant strains proved to have the major portion of the resistance controlled by a single dominant locus or region (Ogaki and Tsukamoto, 1957). Other strains (Crow, 1957) have been shown to have their resistance based on many factors which exert their effects additively. Some of the strains were selected by exposure of adults to aerosols, some to DDT on paper, others by exposure of larvæ to DDT in the food medium, and some by topical application. With this variety of selective methods it is not surprising that a variety of hereditary responses resulted. Furthermore, Levene, Pavlovsky and Dobzhansky (1958) have shown that replicate samples of the same population may react differently under apparently identical conditions.

Insecticide resistance is genetically similar to drug resistance in bacteria, and shares in the continuing controversy over pre-adaptation (the presence in populations of individuals with some degree of hereditary resistance before exposure to the toxic agent) and post-adaptation (the development of resistance by all, or part, of a population by physiological adaptation, which may or may not be inherited, as a result of exposure to the toxic agent). The pre-adaptational view has been well substantiated, for bacteria, by the work of Cavalli-Sforza and Lederberg (1956), the clearest evidence arising from the production of resistance without exposure to the toxic agent by means of sibselection. The best previous evidence in favour of pre-adaptation in Drosophila has been based on the inability of selection of any kind to

* Present address : Department of Biological Sciences, Northern Illinois University, DeKalb, Illinois.

$\uparrow$ Paper number 73 I from the Department of Genetics, University of Wisconsin, Madison 6 , Wisconsin. This work had been supported, in part, by grants from the Office of the Surgcon General, Department of the Army, and from the National Science Foundation. 
produce significant resistance in highly inbred strains (Merrell and Underhill, I956 ; Crow, I957).

The present study was planned to throw light on three main points;

I. If the adaptation is, or can be, a purely selective process, based on hereditary variability already in the population, then it should be possible to select for or against the adaptation without ever exposing the organisms in the direct ancestral line to the selective agent.

2. Will different methods of selection applied to samples of the same starting population give variant results, even with similar culture conditions?

3. The various resistant strains of $D$. melanogaster produced in different laboratories had been selected and tested by different means. Some of these strains were obtained and cultured and tested along with the strains selected in the Madison laboratory. They can now be compared as to degree of resistance under the conditions of the Madison laboratory.

\section{MATERIALS AND METHODS}

The flies were tested for DDT tolerance by being exposed to DDT deposited on paper lining the vials in which they were confined. Papers were prepared with the following concentrations of DDT, in micrograms per square centimetre (10-6 $\left.\mu \mathrm{gm} . / \mathrm{cm}^{2}\right): \mathrm{I} / 25(0.04) ; \mathrm{I} / 5(0 \cdot 20) ; \mathrm{I} ; 5 ; 25 ; \mathrm{I} 25 ; 625$. Some were produced with 6050 for use in the population cages with direct selection.

The DDT-lined vials were assembled into " test sets." In the initial testing, of generations $O$ and $\mathrm{I}$, a " test set" consisted of 5 vials with the concentrations : $\mathrm{I} / 25, \mathrm{I} / 5, \mathrm{I}, 5,25$. The number of vials was later reduced to three, and two different kinds of " test set" were produced, the " high sets" with the concentrations : 1/5; 5 ; and I25 (later I, 25, 625), and the "low sets" with concentrations : I/25; I ; 25 .

The lines selected for resistance to DDT were tested by exposure to the "high sets," those selected for susceptibility to DDT were tested by the "low sets." (Exposure 18 hours, counts 24 hours later.) By using test concentrations in regular multiples it was possible to apply "Karber's Method" (Cornfield and Mantel, I950) to obtain an estimate of the dose which would kill half of the population $\left(\mathrm{LD}_{50}\right)$ represented by the flies tested in each set.

The $\mathrm{LD}_{50}$ values for each test set were treated as units of data in the statistical analysis. In preparing a test from three to six flies were required for each vial. Thus the $\mathrm{LD}_{50}$ value from a particular " test set" was based on the performance of 9 to $\mathrm{I} 8$ flies.

All testing was done on female flies, which give more reproducible results than males (Crow, 1954). In the case of the sib-selected lines it was not practical to use females of selected size, age, and condition because of the very small number of flies available in each culture and the lack of synchrony between cultures. This choice of material undoubtedly increased the variability of the results, but seems realistic in that it involved all adult stages rather than one selected age. All tests of DDT tolerance were made in incubators adjusted to $25^{\circ} \mathrm{C}$.

The test sets were serially numbered and used repeatedly in rotation for several months. Thus each set was used the same number of times and changes in potency were presumed to be proportional to the various sets. Differences between sets were presumed to be equally distributed among all tested strains. 


\section{(i) Methods of selection}

All selections were made on samples of a heterogeneous stock, produced from i 7 genetically distinct laboratory strains and 9 wild type strains derived from 9 females collected at a local fruit warehouse.

Cage I had no exposure to DDT at any time during the course of the experiment. It therefore can be considered a control. Cage 2 had DDT added for lengthy periods, then removed for similar periods. After early intermittent application, similar to Cage 2, Cage 3 had continuous exposure to DDT. The DDT was introduced to the cages on papers identical with those used in the test vials (except that higher concentrations were used). The papers were left in the cages for one or more weeks, being replaced periodically.

\section{(ii) The sib-selection lines}

An initial group of 46 pairs of flies from the heterogeneous stock were placed in a similar number of food vials and allowed to produce progenies. Thus each pair of flies produced one progeny ( $=$ sibship $=$ family) in one food vial. Each progeny was tested for DDT tolerance. After the tests were completed the progenies were ranked according to their tolerance, as evidenced by the group of females tested from each. The parents of the next generation were chosen from the untreated sibs of the tested progeny. The most resistant 5 progenies each provided 4 males and 4 females (mated serially) toward the establishment of the " High" line. The next $\mathrm{i}_{5}$ most resistant progenies each provided 2 males and 2 females (mated serially) to the "High" line. The 20 most susceptible progenies provided similarly for the establishment of the "Low" line. In each case the flies were pair mated, with one pair to each food vial. The result was 50 vials, each with a pair of parents, to produce 50 progenies to be tested in the next generation of each line. The process was repeated for each generation of selection, except that after generation i duplicate sets of parents were made up for each line, with the result that two "High " and two "Low" lines were available for selection. The High and Low lines were carried in pairs through all procedures, thus High Line I $\left(\mathrm{HLI}_{\mathrm{I}}\right)$ and Low Line I ( $\left.\mathrm{LL} \mathrm{I}\right)$ were tested at the same time and were raised on the same batches of food. Similarly High and Low Lines 2 ( $\mathrm{HL}_{2}$ and $\mathrm{LL}_{2}$ ) were carried together.

About half-way through the selection a combination of events, most importantly a series of batches of poor food medium (the nature of the poorness is unknown), led to such a reduction of numbers in all sib-selection lines that mass mating was necessary to avoid losing the lines. This interval was named the "Mass Mating Hiatus," and is so designated on the appropriate figs. The minimum effective population size during this period is unknown, but is estimated to have exceeded 25 in all lines.

\section{RESULTS}

In all cases where selection was practised a change in degree of tolerance of DDT was produced. The amount of change, and the final degree of tolerance, varied considerably.

\section{(i) The population cages}

The data on the population cages are presented in fig. I. Here the tests on the cages are summarised by two-month periods. The fortuitous variation in the values is apparent. Cage 2 seems to vary almost at random, but with a set of values quite different from those of Cage I. These short-term changes almost certainly represent differences in the culturing of the samples taken from the cages, rather than actual changes of the cage populations. The critical point is the final difference 
of tolerance between the cages. Cage 3 shows similar variation but a final greater tolerance to DDT than the other two.

If a period-by-period comparison is made between the three cages it will be noticed that the very high and very low points tend to occur together for all three. This is perhaps the best indication of a common external factor influencing the estimation of DDT tolerance of the three populations.

One may then conclude that in the case of these three cages continuous selection (Cage 3) was more effective than intermittent selection (Cage 2) as one might expect. In both cases an increase of DDT tolerance over that of the control (Cage I) was achieved.

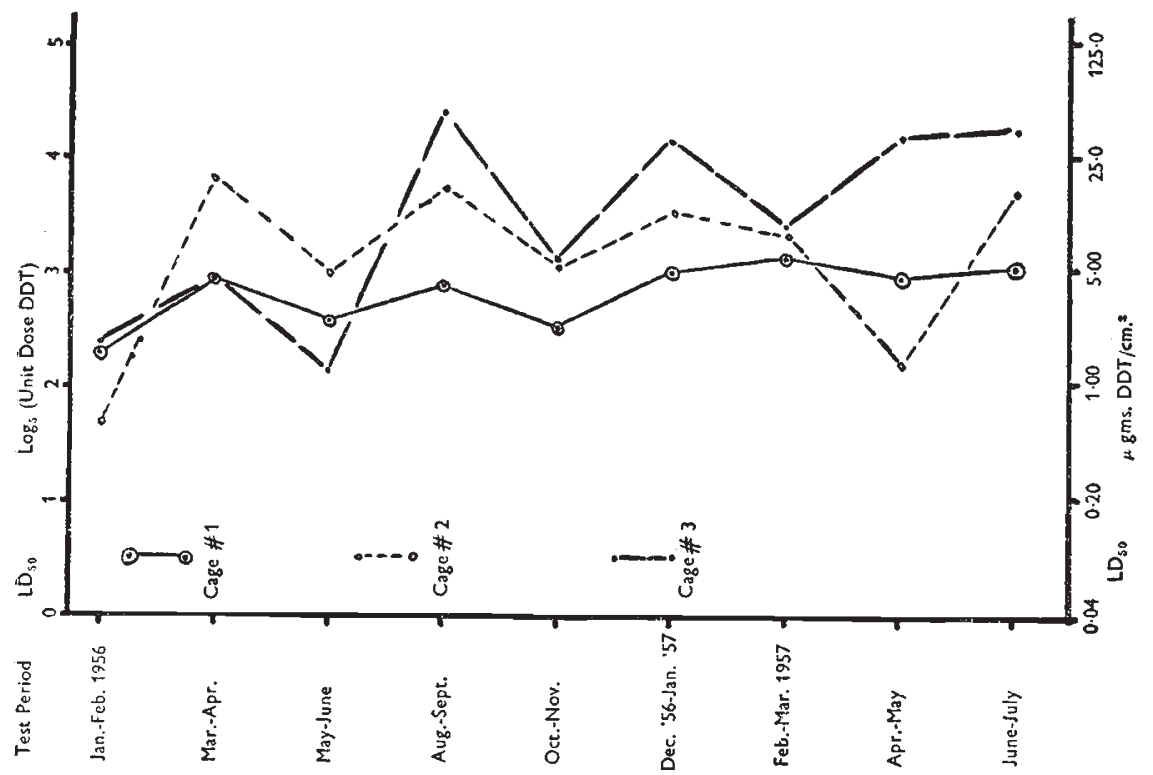

FIG. I.-The comparative DDT tolerance of samples of 3 population cages, \# I unselected, \#2 intermittent selection, \#3 nearly continuous selection.

(ii) The sib-selection lines

The sib-selection lines were carried with selection for I4 or I 5 generations, during the period of time that the population cages were being sampled. Fig. 2 shows clearly that the sib-selection was effective in $\mathrm{HL}_{\mathrm{I}}$ and LLI. For the first three generations there was overlapping, and even reversal of relative positions of the values for the two lines. Then the lines quite clearly separated and remained so, with no hint of overlap at the 95 per cent. confidence level. Most of the difference between the two lines had been achieved by the $5^{\text {th }}$ generation. Only relatively small gains were made later.

Fig. 3 presents a similar picture for $\mathrm{HL}_{2}$ and LL2. These lines were split off from HLI and LLI after generation I. They demonstrate 
quite clearly that similar selective procedures on genetically nearidentical stocks can have very different results. The second lines appear to have achieved most of their separation at a time (both in strict chronology and in generations) when selection had become nearly ineffective in the first lines. The final degree of tolerance or susceptibility to DDT produced in the first and second lines was about the same.

During the selection period various mutant phenotypes appeared repeatedly, both in samples of the population cages and in the sibselection lines. These were all recessives, some sexlinked, and had survived from the origin of the heterogeneous stock.

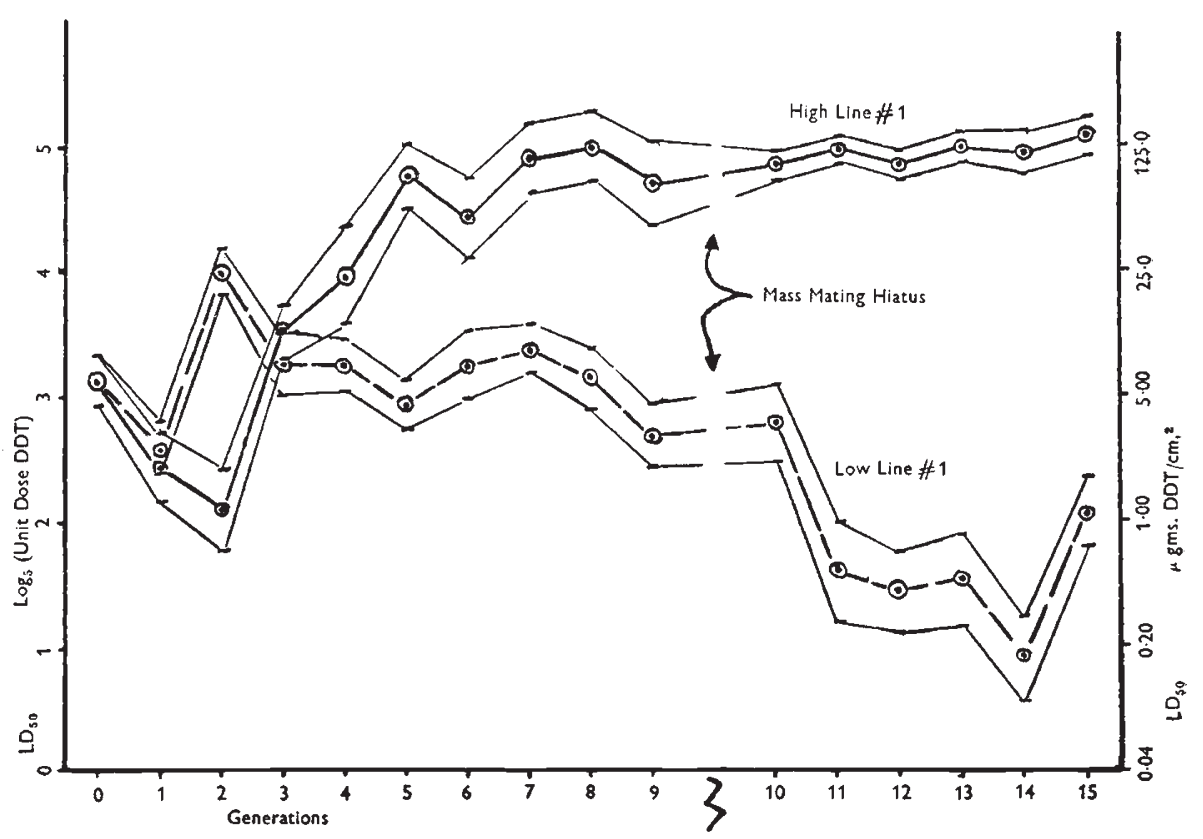

FIG. 2.- The course of selection in High and Low Lines \# I. Mean and 95 per cent. confidence limits of $\mathrm{LD}_{50}$ 's of Sibships in each generation of sib-selection.

At the end of the selection, stocks were made up from the sibselection lines. For each Line two representative stocks, one extreme stock and a variable number of mutant phenotype stocks were prepared. The identity of the mutant phenotypes was not determined.

Fig. 4 presents a comparison of these various stocks derived from the sib-selected lines. It shows that the presence of the recessive mutants is not sufficient to ensure a significantly greater sensitivity to DDT. The very fact of the persistence of these mutants during the course of selection is, of course, evidence that they were not seriously deleterious under the conditions then present.

One could imagine that selection for susceptibility could simply mean selection for general weakness, and that selection for tolerance 
to DDT could mean selection for general fitness. If this were so then one might expect fixation, or high frequency, of many mutants in the Low Lines and elimination of mutants in the High Lines. In fact the High Lines had as many or more mutants (both in kind and frequency) as did the Low Lines. The persistence of these mutants was caused by some selective effect more subtle than simple hardiness.

\section{(iii) Comparison with other stocks}

DDT resistant stocks obtained from other laboratories were tested to provide a comparison with the products of selection described in this study. Fig. 5 shows the results of subjecting all available selected

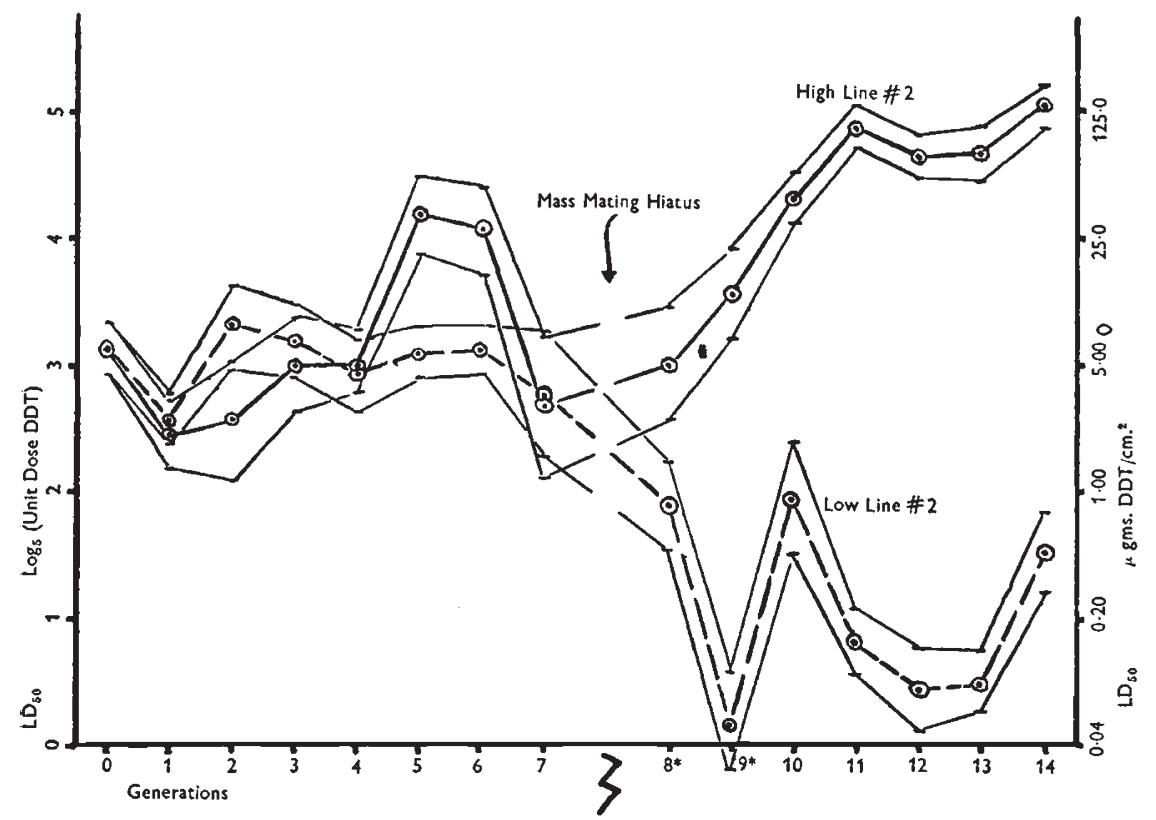

FIg. 3.-The course of selection in High and Low Lines \#2. Mean and 95 per cent. confidence limits of $\mathrm{LD}_{50}$ 's of Sibships in each generation of sib-selection.

* Defective tests, not strictly comparable to the rest.

stocks to the same culture and testing conditions. This comparison was of particular interest because there was doubt as to the relative degree of resistance achieved in different laboratories (i.e., Tsukamoto, r958; Milani, r 957).

It is clear from fig. 5 that the sib-selected Low Lines are much less tolerant of DDT than the unselected Cage I or any others shown, though the unselected $\mathrm{M}-5$; Cy $\mathrm{Pm} ; \mathrm{Sb} / \mathrm{Ubx}^{130}$ stock (not illustrated) was even more sensitive. One might suppose that the Low Lines represent an approach to the maximum sensitivity consistent with good viability and fertility - traits which are not characteristic of the $\mathrm{M}-5 ; \mathrm{Cy} / \mathrm{Pm} ; \mathrm{Sb} / \mathrm{Ubx}^{130}$ stock. 
The values shown for Cages 2 and 3 (in fig. 5) are those of the final two-month test period in each case. These values were chosen because they were obtained at the same time as most of the values for the other stocks. The Hikone-R strain which was selected by DDT food medium from an already resistant wild caught strain (Ogaki and Tsukamoto,

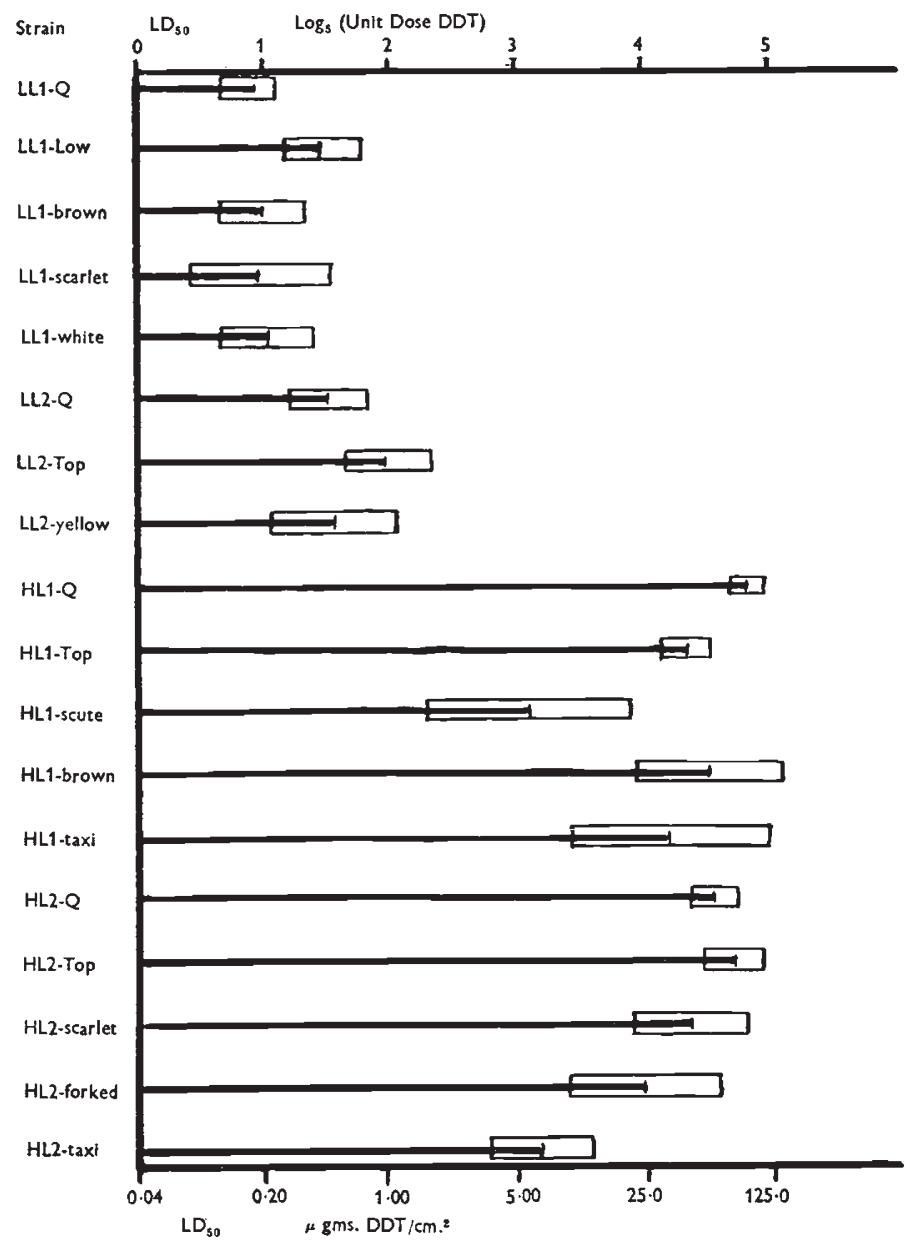

FIG. 4.-A comparison of the results of testing DDT tolerance of a number of strains and sub-strains selected for resistance and susceptibility to DDT. Mean and 95 per cent. confidence limits illustrated. ( $Q$ indicates stock made up from a representative group at the final selected generation. Top indicates stock made up from most favourable 5 progenies of the final selected generation. Low indicates stock made up from most susceptible 5 progenies of the final selected generation. Loci responsible for indicated mutant phenotypes unknown.)

1957) seems to have about the same degree of tolerance to DDT as the High sib-selected Lines. The Brown eye-R stock was selected by Crow and has been maintained in small cultures for a number of years without further selection. Its level of resistances at the time of this 
study was still much above that of the control and intermediate between Cages 2 and 3 .

The stocks SySM-I, SyS-I002, SyS-I02 and ORS-Ioor were selected by King (1954, 1957) using an aerosol containing DDT as the selective agent. All of the selection was direct, survivors of a given treatment were used as parents of the next generation (as was also the case with the Hikone- $R$, Brown eye- $R$, and Cage \#2 and \#3

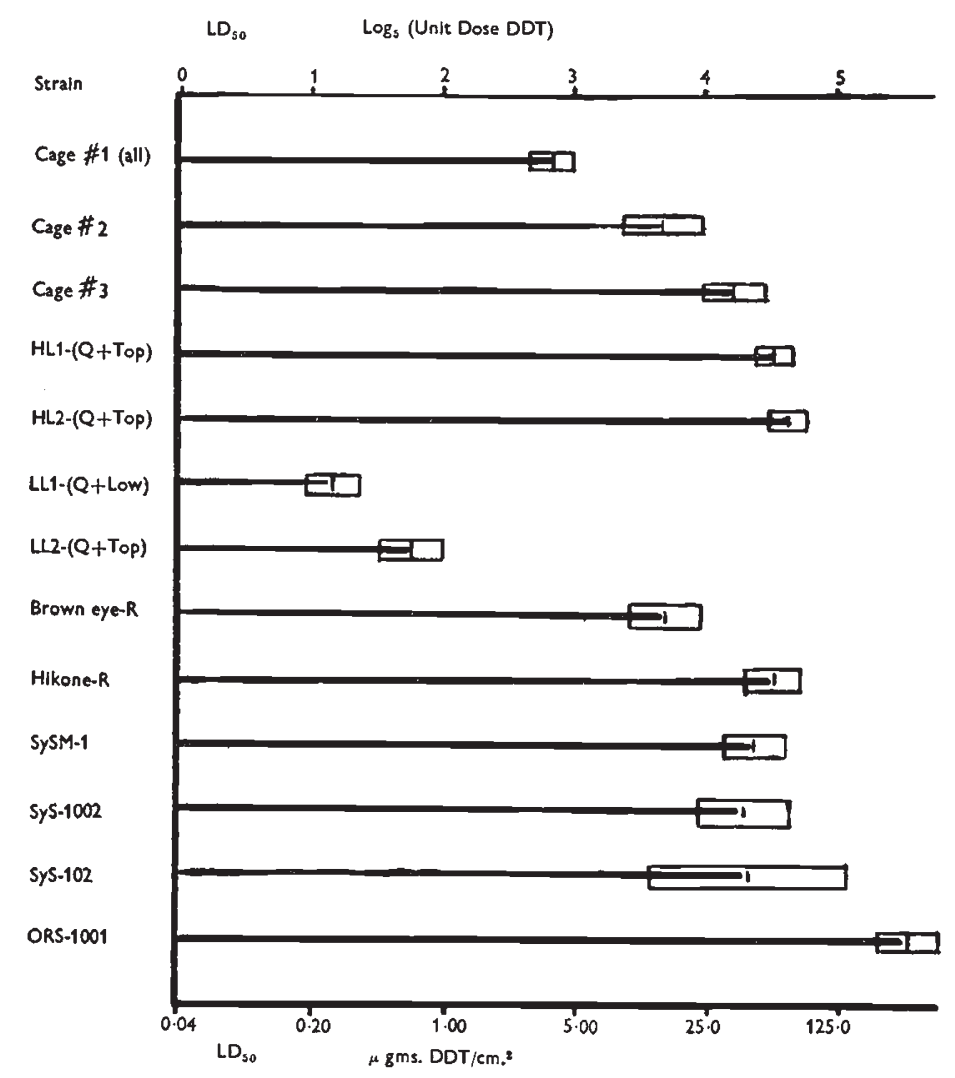

Fig. 5.-A comparison of the results of testing a number of different strains of Drosophila melanogaster Meigen for DDT tolerance. Mean and 95 per cent. confidence limits illustrated. ( $Q+$ Top $),(Q+L o w)$, etc. indicate combined results for non-mutant stocks from indicated selected Line.)

stocks). The effectiveness of the sib-selection is most clearly shown here, where the sib-selection lines are seen to have become as, or more, tolerant of DDT than all of the direct selection stocks, except ORS-Ioo I .

\section{GENETIC BASIS OF RESISTANCE}

The High and Low sib-selected Lines, the population cage stocks, the Brown eye-R, and Hikone-R stocks were outcrossed to $M-5$; $\mathrm{Cy} / \mathrm{Pm} ; \mathrm{Sb} / \mathrm{Ubx}^{130}$ stock and the different phenotypic classes of hybrid offspring were tested. Fig. $6(a$ and $b)$ gives graphic comparison of the results. 
Probably the most obvious point in this comparison is that the Hikone-R resistance factors are clearly dominant over the susceptibility factors in the $\mathrm{M}-5 ; \mathrm{Cy} / \mathrm{Pm} ; \mathrm{Sb} / \mathrm{Ubx}^{130}$ stock. In fact the hybrids seem to show a degree of heterosis, in that some of the phenotypic classes have a higher mean $\mathrm{LD}_{50}$ than did the parental Hikone-R stock.

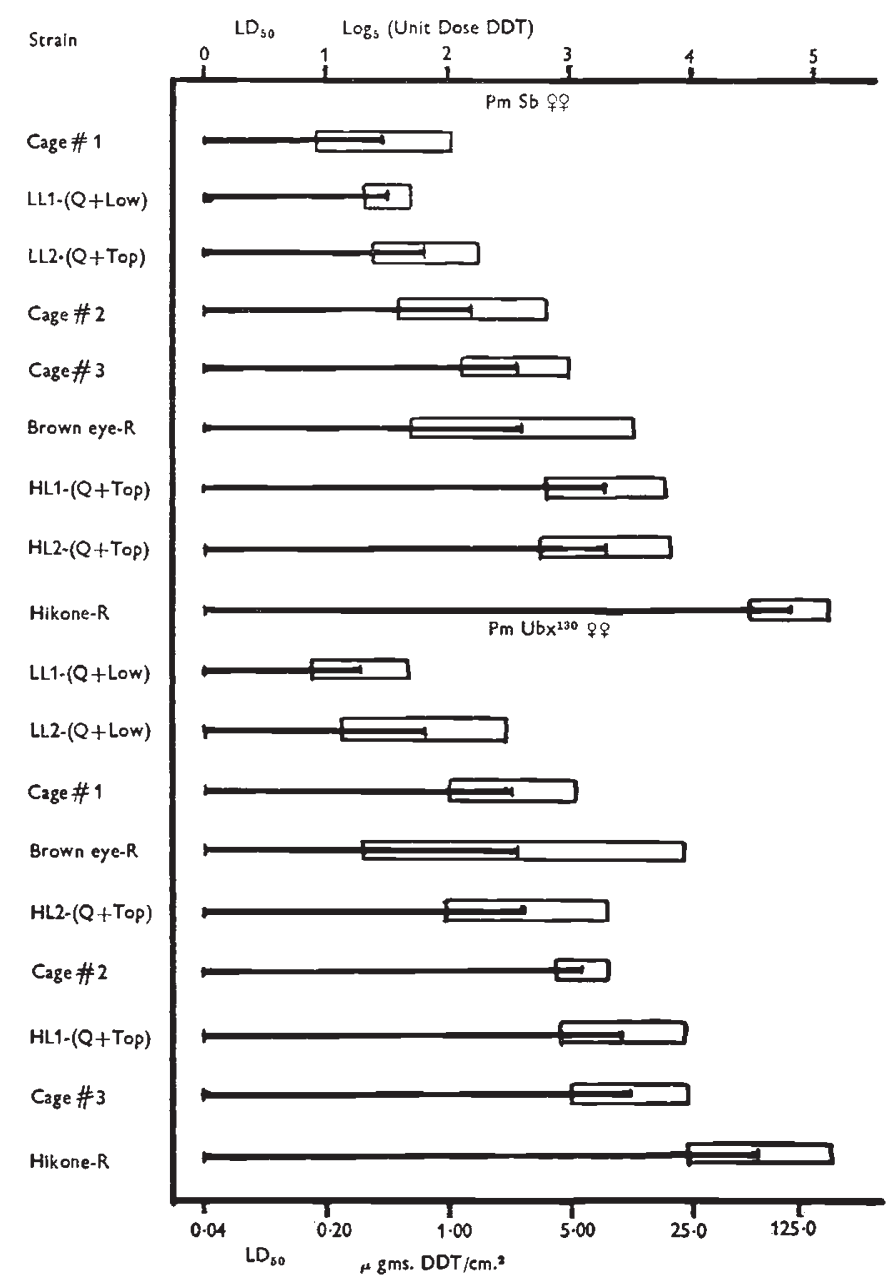

FIG. 6a.-The results of testing $F_{1}$ 우우 offspring of various strains outcrossed to the M-5; $\mathrm{Cy} / \mathrm{Pm}$; Sb/Ubx ${ }^{130}$ stock. Grouped by similar phenotypes (Pm Sb, Pm $\mathrm{Ubx}^{130}$ ). Mean and 95 per cent. confidence limits illustrated.

The comparisons of the other stocks are less clear. The great degree of variation within each group confuses the relative positions. The changes in relative rank, where the difference is significant, between the different phenotypic classes gives evidence of the difference in genetic basis of the resistant stocks. The interaction with the various mutant chromosomes was apparently quite different for many of the stocks. The sib-selected High stocks and the Cage 3 stock show changes in relative position in each phenotypic class. The unselected 
Cage I stock was generally about the same as the Low sib-selected Lines in these outcrosses. Apparently the susceptibility factors were completely recessive and the resulting tolerance was simply a reflection of heterosis between genetically diverse stocks.

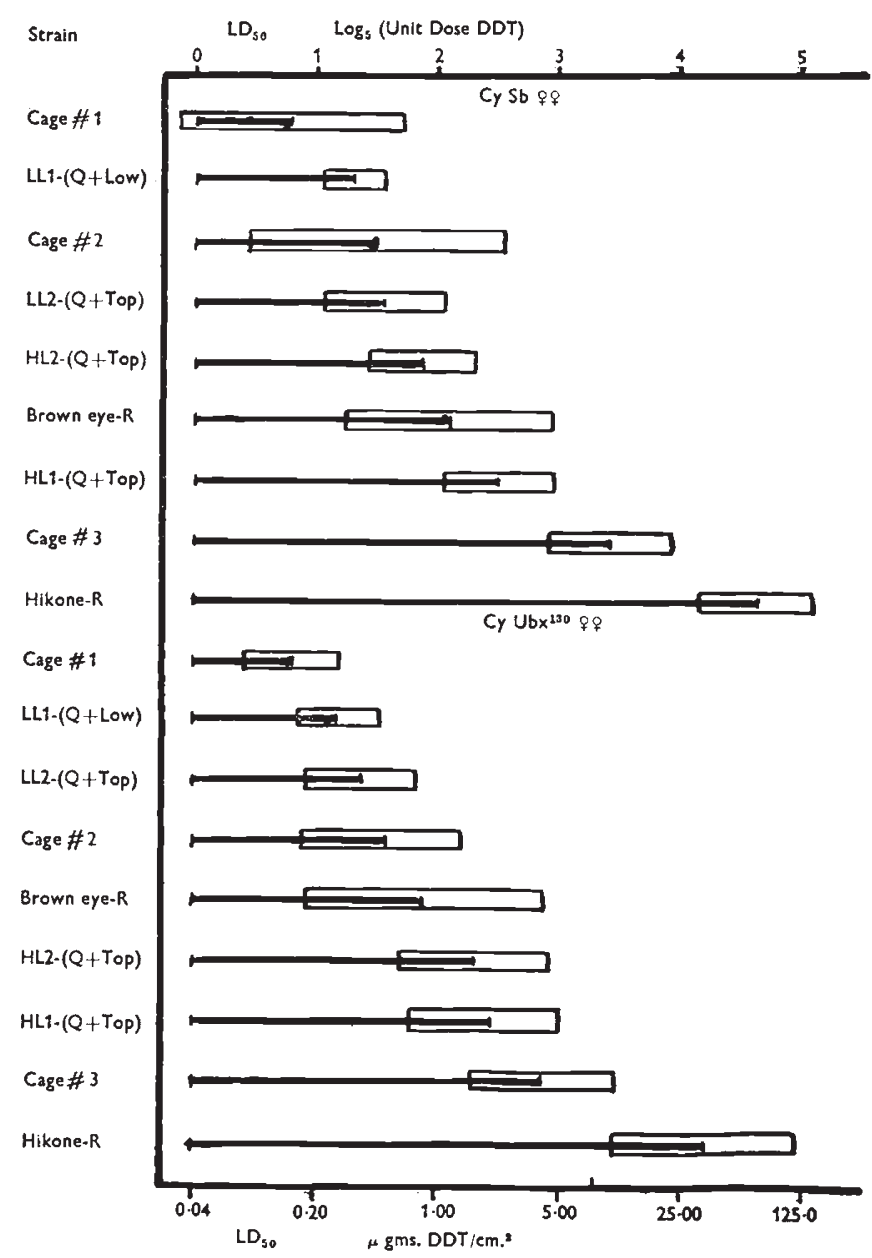

FIG. $6 b$. The results of testing $\mathrm{F}_{1}$ o +9 offspring of various strains outcrossed to the M-5; $\mathrm{Cy} / \mathrm{Pm} ; \mathrm{Sb} / \mathrm{Ubx}^{130}$ stock. Grouped by similar phenotypes (Cy Sb, Cy $\left.\mathrm{Ubx}^{130}\right)$. Mean and 95 per cent. confidence limits illustrated.

\section{DISCUSSION}

It is evident from figs. $\mathrm{I}, 2$, and 3 that both direct and sib-selection have been effective. The plotted curves are very similar, in general, to selection curves relating to quite different characteristics in $D$. melanogaster and other species. In this study the period of rapid change in the populations came at different times in the different lines, but was relatively early when compared with the lines developed by King (r957). Some of King's lines showed little response to selection until 
they had undergone 20 or 30 generations of selection. They then rose rapidly (in resistance) to high values. King's ORS-I oo I finally achieved an $\mathrm{LD}_{50}$ nearly 35 times that of the control line after 73 generations of selection, and his SyS-Ioor and SyS-ıoo2 lines achieved values of $x 6$ and 20 times that of the control lines after some 60 generations. These lines were selected at the 50 per cent. level by direct exposure to DDT aerosols. Their changes compare well with the following values of the difference of the sib-selected lines from their control: HLI, 34x; $\mathrm{HL}_{2}, 35 \mathrm{x} ; \mathrm{LL}$, $\mathrm{I} / 3 \mathrm{x}$; LL2, I/8x; in $\mathrm{I} 5$ generations for the first Lines and 14 generations for the second Lines.

The reason for the shorter selection times for the sib-selection lines can probably be found both in their origin and in the selection method. By sib-selection those progenies from parents capable of producing resistant genotypes were selected, but non-resistant genotypes were also included by the same token. In King's lines only resistant phenotypes survived to reproduce. Thus in the sib-selection lines genes which might, in a different genotypic environment, produce resistance, were kept in the population. In the direct selection in King's lines only strongly resistant phenotypes survived, genes with the mere potentiality to produce resistance in another genotype being quickly eliminated. King's lines originated from sources of relatively little variability, The sib-selection and population cage lines reported here were started with all of the variability obtainable in 26 different sources at hand. With greater initial genetic variability, and a breeding system that tended to maintain variability, it is not too surprising that selection proceeded more rapidly in this study. There appears to be some indication (figs. 3 and 4 ) that the "Mass Mating Hiatus" may have allowed more effective recombination in the sib-selected lines and thus contributed to the post-Hiatus gains made by some of the lines.

The results of the outcrosses to the $\mathrm{M}-5 ; \mathrm{Cy} / \mathrm{Pm} ; \mathrm{Sb} / \mathrm{Ubx}^{130}$ stock indicated that each of the resistant lines had a somewhat different genetic basis, at least as indicated by degree of dominance and interactions with the various tester chromosomes. This result is in agreement with King's (1957) conclusions, and seems to show again, if indeed it need be shown, that populations of similar origin will respond in different ways to similar selective pressures, and can achieve similar phenotypic endpoints with different genotypic bases. The outcrosses also show that the sib-selected high lines appear to show less dominance than the mass-selected Cage 3. This perhaps indicates that sib-selection was more likely to pick out recessives than was the mass selection in a population cage.

Crow ( 1957 ) has proposed that in a large randomly mating population selection would be strongly in favour of genes which conferred good general viability and a maximum average resistance in a variety of genotypic backgrounds. The sib-selection lines were selected primarily for resistance, and for viability only to the extent of being able to produce a minimum of 20 to 25 females, in each progeny. 
Since the sib-selection lines were relatively small in size-only 25 effective pairs of parents in each generation (thus a maximum of roo samples of each chromosome) - there would be greater likelihood of selecting chromosomes which had favourable specific interactions. Some support for the view that the selection in the sib-selected lines was not strongly for general fitness is obtained by comparing the values of resistance of each of the High Lines in the final selected generation to their mean value during the following two months, when they were mass cultured and tested, but not selected. High Line I had a final selection value for the $\mathrm{LD}_{50}$ of 133.5 , but a mean value for the following two months of $57 \cdot 9$. High Line 2 had similar values of $137 \cdot 0$ and $67 \cdot 8$. These represent a loss of about one-half of the resistance, on the average, during this period. The fact that the lines still retained a resistance higher than Cage 3 and most of the other lines tested may reflect simply the short time available for reversion to have occurred. However, the loss may only reflect the difference of culture conditions between the relatively uncrowded vials, during sib-selection, and crowded bottles during the later period.

By sib-selection it has been possible to show that a single population can contain the genetic factors for either sensitivity or resistance to an environmental agent, and that those factors can be selected and concentrated without contact with the agent. This proof of preadaptation in this case does not of course eliminate the possibility of post-adaptation under some other circumstances. The proof does, however, make the hypothesis of post-adaptation superfluous.

It has been shown by Lüers (I953) that DDT is not mutagenic in D. melanogaster, thus eliminating the possibility that direct exposure might produce mutants, some of which might contribute to a postadaptation. This is supported also by the fact that the directly selected lines were generally inferior to the sib-selected lines in resistance, which would hardly be expected if exposure to DDT were producing mutations that contributed to the resistance. Crow (unpublished) has shown that doses of DDT which do not kill, will not select for, or in any way increase, resistance. Thus for this species, as for some bacteria (Cavalli-Sforza and Lederberg, I956), it is not necessary to propose any mechanism for the appearance of resistance in populations other than selection of pre-existing variation normally present in those populations.

\section{SUMMARY}

A heterogeneous stock of Drosophila melanogaster Meigen was. proliferated in population cages and sub-populations extracted for selection. Samples of each of an initial group of 46 pair mating progenies were tested in vials lined with DDT impregnated paper to determine resistance to kill. The untested sibs from the 20 most resistant and the 20 least resistant progenies were used to make up $5^{\circ}$ pair matings to start each of the prospective lines. Similar testing and selection was carried out on subsequent generations for each line. 
Selection was carried on for 15 generations. At the end of this period the median lethal doses for the resistant lines were approximately r 25 and 625 times as great as those for their respective paired susceptible lines, and more than 30 times as great as the median lethal dose for the control stock. Two population cages were subjected to direct contact with DDT and tested during the same period. The resistance developed by sib-selection compares favourably with the resistance of lines, from this and other laboratories, that were produced by direct selection. The pre-adaptation hypothesis is sufficient to account for the resistance of the sib-selected lines.

Acknowledgment.-The author wishes to express thanks to Dr J. F. Crow, for suggesting this problem, for many discussions of the work and results, and for making his laboratory available.

\section{REFERENCES}

BRown, A. W. A. 1958. Insecticide resistance in arthropods. World Health Org., Geneva, 1958.

CAVALLI-SFORZA, L. L., AND LEDERBERG, J. I956. Isolation of pre-adaptive mutants in bacteria by sib-selection. Genetics, $41,367-381$.

CORNFIELD, J., AND MANTEL, N. I950. Some new aspects of the application of maximum likelihood to calculation of the dosage response curve. $\mathcal{F}$. Am. Stat. Assoc., 45, I8 1-2 I 0 .

CRow, J. F. I954. Analysis of a DDT-resistant strain of Drosophila. F. Econ. Entomology, 47, 393-398.

CRow, J. F. 1957. Genetics of insect resistance to chemicals. Ann. Rev. Entomology, $2,227-246$.

KING, J. C. I954. The genetics of resistance to DDT in Drosophila melanogaster. 7. Econ. Entomology, 47, 387-393.

KING, J. C. 1957. Investigation of the genetic nature of resistance to insecticides developed by populations of Drosophila melanogaster. Final report of res. by Long Island Biol. Assoc. for Med. Res. and Dev. Board, Off. Surgeon General, Dept. of the Army, U.S.A.

LeVene, H., PAVlovsky, O., AND DobZhansky, Th. 1958. Dependence of the adaptive values of certain genotypes in Drosophila pseudoobscura on the composition of the gene pool. Evolution, I2, I8-23.

LÜERS, H. I953. Untersuchung zur frage der mutangenität des kontaktinsektizids DDT an Drosophila melanogaster. Naturwiss, ro, 293.

MERRELL, D. J., AND UNDERHILl, J. C. I956. Selection for DDT resistance in inbred, laboratory, and wild stocks of Drosophila melanogaster. F. Econ. Entomology, 49, 300-306.

MILANI, R. I957. Genetic Research on the resistance of insects to the action of toxic substances. WHO, Div. of Environ. Sanit. Inf. Cir. on the Resistance Prob. Suppl. C-Oct. 1957. (English translation of Rivista di Parasitologia, 17, 233-246 (1956) and $18,43-60$ (1957)).

ogAKI, M., AND TSUKAMOTO, M. I957. Genetical analysis of DDT resistance in some Japanese strains of Drosophila melanogaster. In : Fapanese Contribution to the Study of the Insecticide Resistance Problem. Brown, Takei, Nagesawa, Editors. Publ. by the Inst. of Insect Control, Kyoto Univ. for the World Health Organisation, 1957, pp. 28-32. (English version of "Botyu-Kagaku", I8, I OO-I O4 (I953)).

тsuкamoto, м. 1958. Genetics of insecticide-resistance in Drosophila. In: World Health Organisation, Sem. on the resistance of insects to insecticides, World Health Organisation and the Government of India, New Delhi : 27 Feb.-7 Mar. 1958, pp. 69-75. (Unpubl. working Doc. WHO/Insecticides/76) Issued : April I958. 